\title{
MEAN FLOW INDUCED BY THE VISCOUS CRITICAL LAYER IN A STRATIFIED FLUID
}

\author{
NOEL SMYTH ${ }^{1}$
}

(Received 6 September 1985; revised 3 March 1986 and 16 December 1986)

\begin{abstract}
The evolution of the critical layer in a viscous, stratified fluid is examined in the limit of large Richardson and Reynolds numbers. A source far above the critical layer and of amplitude $\varepsilon$ is turned on at $t=0$ and the behaviour of both the steady state and transients is found. Viscosity dominates over nonlinearity in the critical layer for $\varepsilon \sqrt{\operatorname{Re}} \ll 1$, Re being an appropriately defined Reynolds number. Wave amplitudes are found to grow as the critical layer is approached, then decay rapidly due to the action of viscosity in a critical layer of $\left.\mathrm{O}(\mathrm{Re})^{-1 / 3}\right)$ around the critical level. The critical layer acts as a source of vorticity, which diffuses into the outer flow, resulting in an induced mean flow of $O\left(\varepsilon^{2} \sqrt{R e} \sqrt{t}\right)$. This induced mean flow causes the critical level to move towards the incoming wave.
\end{abstract}

\section{Introduction}

The interaction of an internal gravity wave with a shear flow has received considerable attention in recent years. The major feature of the interaction is the so-called critical level, where the shear velocity and the wave velocity in the direction of the shear are equal. Linear theory predicts a singularity at this critical level as wave action piles up at this level due to the vanishing of the group velocity there. Interest has centred on how invoking nonlinearity and/or viscosity smooths out this singularity.

\footnotetext{
${ }^{1}$ Department of Applied Mathematics, University of New South Wales, P.O. Box 1, Kensington, N.S.W., Australia, 2033

(C) Copyright Australian Mathematical Society 1988, Serial-fee code 0334-2700/88
} 
The critical level is of great geophysical significance as it acts as a barrier to wave propagation and causes vertically propagating energy to propagate horizontally. Furthermore, convective instabilities at the critical level, due to the reduction of the local Richardson number below zero, result in turbulent mixing.

Booker and Bretherton [2], by considering the linear problem and matching around the critical level, showed that as a critical level is approached, the vertical group velocity goes to zero and the wave is effectively absorbed. At the critical level, the amplitude of the wave is attenuated by a factor

$$
f=\exp \left[-\pi\left(J_{0}-\frac{1}{4}\right)^{1 / 2}\right],
$$

where $J_{0}$ is the Richardson number at the critical level.

As the critical level is approached however, the amplitude of the horizontal velocity becomes large and the validity of linear theory is questionable. To overcome this deficiency, viscosity and/or nonlinearity must be invoked near the critical level. Either of these two effects will limit the magnitude of the horizontal velocity. Which of these effects dominates will depend on the particular physical situation.

Grimshaw [11], [12] considered the flow in the large Richardson number limit, in which case the appropriate theory is that for slowly varying waves. The governing equations in this limit were derived, for arbitrary initial amplitude, by the method of multiple scales. These equations are essentially the wave action equation and an equation for the wave-induced mean flow. The behaviour of a wave packet as it propagated toward a critical level was examined. It was found that the amplitude of the wave grew to a maximum as it approached the critical level, then rapidly decreased to zero due to viscosity. The passage of the wave was found to result in a permanent acceleration of the mean flow.

Dunkerton [7], [8] also used the same slowly varying approximation, with the further assumption that the wave frequency was constant. Similar results were found to those of Grimshaw [12]. Dunkerton and Fritts [9] and Fritts and Dunkerton [10] subsequently extended the scope of the slowly varying approximation by numerically solving the governing equations.

Brown and Stewartson [4], [5], [6] invoked nonlinearity rather than viscosity in the critical layer. The evolution of the waves produced by a steady harmonic forcing (switched on at $t=0$ ) far above the critical level was examined. To reduce the algebraic complexity, the latter two papers considered the large Richardson number limit. It was found that both the steady state wave and one of the transients must be considered in the critical layer, as both these waves are singular near the critical layer. The interaction between these waves and their harmonics led to very weak reflected and transmitted waves being generated. The mean flow played a passive role in the interaction process. 
In the present work, we shall consider the same physical situation as that of Brown and Stewartson, except that viscosity rather than nonlinearity will be assumed to dominate in the critical layer. We consider a shear layer separating two parallel streams in a stratified fluid for which the Richardson and Reynolds numbers are large. A source at $y_{0}, y_{0} \gg 1$, whose $x$-dependence is proportional to $e^{i \alpha x}$, and amplitude is $\varepsilon, \varepsilon \ll 1$, is switched on at $t=0$. The solution is constructed using singuiar perturbation analysis based on the large Reynolds number. The behaviour of both the transients and the steady state in both the outer flow and the critical layer will be found. The assumption of a viscositydominated critical layer will be shown to be valid for $\varepsilon \sqrt{R e} \ll 1$, where Re is an appropriately defined Reynolds number.

The elucidation of the mean flow induced by a viscosity-dominated critical layer is a major aim of the present work. By averaging the governing equations, the equations for the induced mean flow are obtained. The critical layer acts as a source of vorticity, which diffuses into the outer flow, resulting in an induced mean flow there. The induced mean flow is of $O\left(\varepsilon^{2} \sqrt{\operatorname{Re}} \sqrt{t}\right)$. This induced flow results in the critical level being moved towards the incoming wave. Grimshaw [13] showed that inviscid theory also predicts that the induced mean flow results in the critical level being moved towards the incoming wave. This was also shown by the numerical work of Dunkerton and Fritts [9] and Fritts and Dunkerton [10]. Both nonlinearity and viscosity dominated critical layers result in the critical level being moved towards the incoming wave.

Brown, Rosen and Maslowe [3] considered a similar situation to that considered here for Richardson number $\frac{1}{4}$ (so that the flow is marginally stable) and Prandtl number 1, as in this case the governing equations have an exact solution for a hyperbolic tangent shear profile. It will be found that the effect of the critical layer on the mean flow in this case is similar to that in the large Richardson number, infinite Prandtl number case considered here.

\section{Equations of motion}

The governing equations to be used in this study are the same as those of Brown and Stewartson [5], except that the viscosity and thermal diffusivity will be assumed to be non-zero. The physical situation is as follows. A shear layer is assumed to separate two parallel streams of fluid. Orthogonal cartesian coordinates moving with the mean fluid velocity are chosen with the $x^{*}$ axis parallel to the two main streams. The equation of state for the fluid is taken to be

$$
\rho^{*}=\rho_{0}^{*}\left[1-\beta^{*} T_{0}^{*}\left(\left(T^{*} / T_{0}^{*}\right)-1\right)\right],
$$




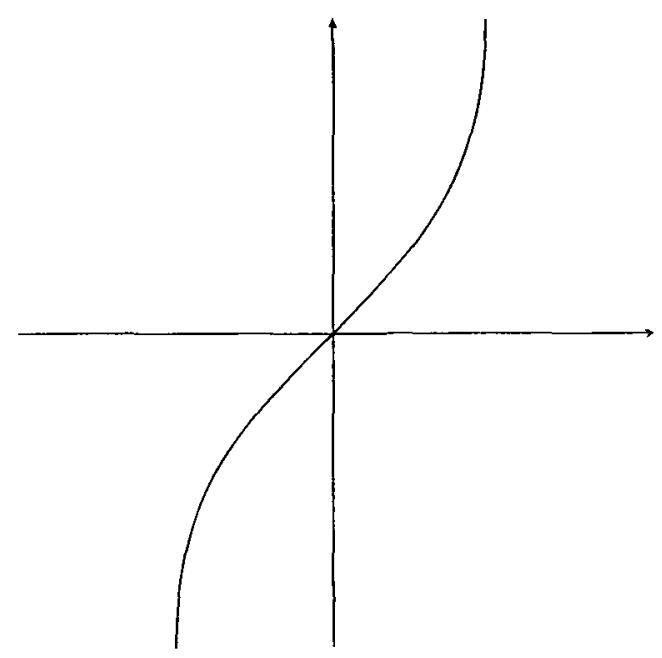

Figure 1. Typical velocity profile $U(y)$.

where asterisks denote dimensional quantities. The density is denoted by $\rho^{*}$, and the temperature by $T^{*}$. $T_{0}^{*}$ is a reference temperature and $\rho_{0}^{*}$ is a reference density.

The Navier-Stokes equations become

$$
\begin{gathered}
\nabla \cdot \mathbf{u}^{*}=0, \\
\mathbf{u}_{\mathbf{t}^{*}}^{*}+\mathbf{u}^{*} \cdot \nabla \mathbf{u}^{*}=-\frac{1}{\rho_{0}} \nabla p^{*}+\beta^{*} g\left(T^{*}-T_{0}^{*}\right) \nabla y^{*}+\nu \nabla^{2} \mathbf{u}^{*}, \\
T_{t^{*}}^{*}+\mathbf{u}^{*} \cdot \nabla T^{*}=\kappa \nabla^{2} T^{*},
\end{gathered}
$$

when the Boussinesq approximation is used. Here $\mathbf{u}^{*}$ is the fluid velocity, $\nu$ is the kinematic viscosity and $\kappa$ is the thermal diffusivity.

To put these equations into non-dimensional form, the reference speed $V^{*}$ is chosen to be half of the velocity difference between the two streams, and the reference length $L^{*}$ is chosen to be an appropriate length scale of the undisturbed flow. Non-dimensional variables are then defined by

$$
\begin{aligned}
x & =x^{*} / L^{*}, \quad y=y^{*} / L^{*}, \quad t=t^{*} V^{*} / L^{*}, \quad \rho=-\beta T_{0}^{*} T, \\
\mathbf{u}^{*} / V^{*} & =U(y) \nabla x+\varepsilon \mathbf{u}(x, y, t), \\
\rho^{*} / \rho_{0}^{*} & =1-\beta T_{0}^{*} R(y)+\varepsilon \rho(x, y, t) .
\end{aligned}
$$

The parameter $\varepsilon$ is a measure of the amplitude of the forcing at $y_{0}, y_{0} \gg 1$. The non-dimensional undisturbed velocity and density of the fluid are $U(y)$ and $1-\beta T_{0}^{*} R(y)$ ( $R$ is chosen in this way so that $R^{\prime}(y)>0$ for stable stratification). By the above non-dimensionalisation, $U(y) \rightarrow \pm 1$ as $y \rightarrow \pm \infty$. We shall assume that $U^{\prime}(y) \rightarrow 0$ as $y \rightarrow \pm \infty, U^{\prime}(y)>0$ for $y$ finite and near zero and $R^{\prime}(y) \rightarrow$ constant as $y \rightarrow \pm \infty$. A typical velocity profile is sketched in Figure 1. 
Defining a perturbation streamfunction $\psi(x, y, t)$ by

$$
\mathbf{u}=(u, v)=\left(\frac{\partial \psi}{\partial y},-\frac{\partial \psi}{\partial x}\right)
$$

and eliminating the pressure leads to the non-dimensional equations of motion

$$
\begin{gathered}
\left(\frac{\partial}{\partial t}+U(y) \frac{\partial}{\partial x}\right) \nabla^{2} \psi-U^{\prime \prime}(y) \psi_{x}-\varepsilon \frac{\partial\left(\psi, \nabla^{2} \psi\right)}{\partial(x, \ddot{y})}=-J T_{x}+(\mathrm{Re})^{-1} \nabla^{4} \psi \\
\left(\frac{\partial}{\partial t}+U(y) \frac{\partial}{\partial x}\right) T-R^{\prime}(y) \psi_{x}-\varepsilon \frac{\partial(\psi, T)}{\partial(x, y)}=\mathrm{Pe}^{-1} \nabla^{2} T
\end{gathered}
$$

The Richardson number $J$ is

$$
J=\beta T_{0}^{*} g L^{*} / V^{* 2}
$$

and the Reynolds and Peclet numbers are

$$
\operatorname{Re}=V^{*} L^{*} / \nu, \quad \mathrm{Pe}=V^{*} L^{*} / \kappa
$$

respectively. It will be assumed in this paper that $J \gg 1, \varepsilon \ll 1$, Re $\gg 1$ and $\mathrm{Pe} \gg 1$. Furthermore, it will be assumed that in the critical layer, viscosity dominates over nonlinearity. The precise conditions on $\varepsilon$ and Re for this to hold will be found later.

It is supposed that the incident wave is generated by a forcing proportional to $e^{i \alpha x}$ at $y=y_{0}, y_{0} \gg 1$, which is switched on at $t=0$ and rises to a constant amplitude as $t \rightarrow \infty$.

\section{Linear solution outside critical layer}

It will be assumed that the incoming wave from infinity is of small amplitude $\varepsilon$ and that viscosity dominates over nonlinearity in the resulting critical layer. The governing equations are then the linear limit of (2.5) and (2.6). Also, as the forcing function at $y=y_{0}$ has $x$-dependence of the form $e^{i \alpha x}$, we set

$$
\begin{aligned}
& \psi=\Psi(y, t) e^{i \alpha x}+\text { c.c. } \\
& T=\theta(y, t) e^{i \alpha x}+\text { c.c. }
\end{aligned}
$$

where c.c. denotes complex conjugate. From (2.5) and (2.6), the equations satisfied by $\Psi$ and $\theta$ are

$$
\begin{gathered}
\left(\frac{\partial}{\partial t}+i \alpha U\right)\left(-\alpha^{2} \Psi+\Psi_{y y}\right)-i \alpha U^{\prime \prime} \Psi+i \alpha J \theta \\
=(\mathrm{Re})^{-1}\left(\frac{\partial^{4} \Psi}{\partial y^{4}}-2 \alpha^{2} \frac{\partial^{2} \Psi}{\partial y^{2}}+\alpha^{4} \Psi\right) \\
\theta_{t}+i \alpha U \theta-i \alpha R^{\prime} \Psi=\mathrm{Pe}^{-1}\left(\theta_{y y}-\alpha^{2} \theta\right) .
\end{gathered}
$$


This system of equations will be solved using Laplace transforms. The Laplace transforms of $\Psi$ and $\theta$ are defined by

$$
\begin{gathered}
\bar{\Psi}(y, s)=\int_{0}^{\infty} e^{-s t} \Psi(y, t) d t, \\
\bar{\theta}(y, s)=\int_{0}^{\infty} e^{-s t} \theta(y, t) d t .
\end{gathered}
$$

Taking Laplace transforms of the governing equations (3.2) and (3.3) gives, upon noting that $\psi=\psi_{t}=0$ at $t=0$,

$$
\begin{gathered}
(i \alpha U+s) \bar{\Psi}_{y y}-\left(\alpha^{2} s+i \alpha^{3} U+i \alpha U^{\prime \prime}\right) \bar{\Psi}+i \alpha J \bar{\theta}=(\mathrm{Re})^{-1}\left(\alpha^{4} \bar{\Psi}-2 \alpha^{2} \bar{\Psi}_{y y}+\partial^{4} \bar{\Psi} / \partial y^{4}\right), \\
(s+i \alpha U) \bar{\theta}-i \alpha R^{\prime} \bar{\Psi}=\mathrm{Pe}^{-1}\left(\bar{\theta}_{y y}-\alpha^{2} \bar{\theta}\right) .
\end{gathered}
$$

Eliminating $\bar{\theta}$ between these equations results in

$$
\begin{gathered}
(\mathrm{Re})^{-1} \partial^{6} \bar{\Psi} / \partial y^{6}-\left[3 \alpha^{2} \operatorname{Re}^{-1}+\left(1+\operatorname{Re}^{-1} \mathrm{Pe}\right)(s+i \alpha U)\right] \partial^{4} \bar{\Psi} / \partial y^{4} \\
-2 i \alpha U^{\prime} \partial^{3} \bar{\Psi} / \partial y^{3}+\left[3 \alpha^{4} \operatorname{Re}^{-1}+2 \alpha^{2}\left(1+\operatorname{Re}^{-1} \mathrm{Pe}\right)(s+i \alpha U)\right. \\
\left.+\operatorname{Pe}(s+i \alpha U)^{2}\right] \partial^{2} \bar{\Psi} / \partial y^{2}+2 i \alpha\left(U^{\prime \prime \prime}+\alpha^{2} U^{\prime}\right) \partial \bar{\Psi} / \partial y \quad(3.6) \\
-\left[\alpha^{6} \operatorname{Re}^{-1}+\alpha^{4}\left(1+\operatorname{Re}^{-1} \operatorname{Pe}\right)(s+i \alpha U)+\alpha^{2} \operatorname{Pe}(s+i \alpha U)^{2}\right. \\
\left.+i \alpha U^{\prime \prime} \operatorname{Pe}(s+i \alpha U)-i \alpha U^{\prime \prime}+\alpha^{2} \operatorname{Pe} J R^{\prime}\right] \bar{\Psi}=0 .
\end{gathered}
$$

This equation can be solved by the standard techniques of singular perturbation theory, as Re $\gg 1$ (and $\mathrm{Pe} \gg 1$ ) by hypothesis.

Outside the critical layer, the derivatives of $\bar{\Psi}$ are $O(1)$ and the outer solution in this region may be expanded as

$$
\begin{aligned}
\bar{\Psi} & =\bar{\Psi}_{0}(y, s)+(\operatorname{Re})^{-1} \bar{\Psi}_{1}(y, s)+\cdots \\
\bar{\theta} & =\bar{\theta}_{0}(y, s)+(\operatorname{Re})^{-1} \bar{\theta}_{1}(y, s)+\cdots .
\end{aligned}
$$

The leading order equation in the outer region is, from (3.6),

$$
(s+i \alpha U) \bar{\Psi}_{0 y y}-\left(i \alpha U^{\prime \prime}+i \alpha^{3} U+\alpha^{2} s+\frac{J R^{\prime} \alpha^{2}}{s+i \alpha U}\right) \bar{\Psi}_{0}=0
$$

The flow in the outer region is inviscid and (3.8) is the Laplace transform of the Taylor-Goldstein equation.

The assumption that $J \gg 1$ will now be invoked. For $J \gg 1$, equation (3.8) has the W.K.B. solution

$$
\bar{\Psi}_{0}=C(s) \sqrt{s+i \alpha U(y)} e^{\sqrt{J}} \chi /\left(R^{\prime}(y)\right)^{1 / 4},
$$

where

$$
\chi=-\int_{y}^{y_{0}} \frac{\alpha \sqrt{R^{\prime}(\xi)}}{s+i \alpha U(\xi)} d \xi
$$


The function $C(s)$ is determined by matching with the forcing at $y_{0}$. We find that as $y \rightarrow y_{0}$,

$$
\bar{\Psi}_{0} \sim \frac{C(s) \sqrt{s+i \alpha U\left(y_{0}\right)}}{\left(R^{\prime}\left(y_{0}\right)\right)^{1 / 4}} \exp \left[-\frac{\alpha \sqrt{J R^{\prime}\left(y_{0}\right)}\left(y_{0}-y\right)}{s+i \alpha U\left(y_{0}\right)}\right] .
$$

For this solution to match with the forcing at $y_{0}$, we see that

$$
C(s)=\left(R^{\prime}\left(y_{0}\right)\right)^{1 / 4} F(s) / \sqrt{s+i \alpha U\left(y_{0}\right)} .
$$

The function $F(s)$ is due to the forcing at $y_{0}$ and is such that $F(s) \rightarrow 0$ very rapidly as $|s| \rightarrow \infty$, and $s F(s)$ has a non-zero limit as $s \rightarrow 0$. Therefore the outer solution to leading order is

$$
\begin{aligned}
\Psi_{0}= & \frac{1}{2 \pi i} \int_{\gamma-i \infty}^{\gamma+\infty}\left(\frac{R^{\prime}\left(y_{0}\right)}{R^{\prime}(y)}\right)^{1 / 4} \sqrt{\frac{s+i \alpha U(y)}{s+i \alpha U\left(y_{0}\right)}} \\
& \times F(s) \exp \left\{s t-\int_{y}^{y_{0}} \frac{\alpha \sqrt{J R^{\prime}(\xi)}}{s+i \alpha U(\xi)} d \xi\right\} d s
\end{aligned}
$$

where $\gamma>0$.

To enable the physical significance of this integral representation for $\Psi_{0}$ to be seen, the contour of integration will be rotated by $\pi / 2$. We then find

$$
\begin{aligned}
\Psi_{0}=\frac{1}{2 \pi} & \int_{-\infty+i \gamma}^{\infty+\imath \gamma}\left(\frac{R^{\prime}\left(y_{0}\right)}{R^{\prime}(y)}\right)^{1 / 4} \sqrt{\frac{\sigma-\alpha U(y)}{\sigma-\alpha U\left(y_{0}\right)}} \\
& \times F(-i \sigma) \exp \left\{-i \sigma t-\int_{y}^{y_{0}} \frac{\alpha \sqrt{J R^{\prime}(\xi)} d \xi}{\sigma-\alpha U(\xi)}\right\} d \sigma .
\end{aligned}
$$

The integrand is of the form

$$
I=A(y, \sigma) \exp [i \theta(y, \sigma)],
$$

which is a wave of amplitude $A$, phase $\theta$, with the wavenumber and frequency defined by

$$
m=\theta_{y}=\frac{\alpha \sqrt{J R^{\prime}(y)}}{\sigma-\alpha U(y)}, \quad \omega=-\theta_{t}=\sigma
$$

respectively. Since the Richardson number $J \gg 1$, the integrand (3.15) can be interpreted as a slowly varying wave. The integral representation (3.14) is a superposition of waves with frequency $\sigma$ and wavenumber $m$, each satisfying the internal gravity wave dispersion relation,

$$
(\omega-\alpha U)^{2}=\alpha^{2} J R^{\prime}(y) / m^{2} .
$$

The group velocity $c_{g}$ of the waves is

$$
c_{g}=\frac{\partial \omega}{\partial m}=-\frac{(\omega-\alpha U)}{m}
$$

and is negative, as required. 
The amplitude $A$ of the waves is given by

$$
A^{2}=\left(\frac{R^{\prime}\left(y_{0}\right)}{R^{\prime}(y)}\right)^{1 / 2}\left(\frac{\sigma-\alpha U(y)}{\sigma-\alpha U\left(y_{0}\right)}\right)|F(-i \sigma)|^{2} .
$$

Grimshaw [11], [12] considered the behaviour of a wave packet in a shear flow with a critical level for the case in which the fluid is slowly varying relative to the wave. This slowly varying assumption is equivalent to $J \gg 1$. It was shown that the wave action $A$ satisfies

$$
\frac{\partial A}{\partial t}+\frac{\partial}{\partial y}\left(c_{g} A\right)=0
$$

where

$$
A=\frac{\mathcal{E}}{\omega-\alpha U}, \quad \mathcal{E} \propto \frac{R^{\prime}(y) A^{2}}{(\omega-\alpha U)^{2}} .
$$

On using the expressions (3.16) and (3.18), we find that

$$
c_{g} A \propto \sqrt{R^{\prime}\left(y_{0}\right)}|F(-i \omega)|^{2} / \alpha \sqrt{J\left(\omega-\alpha U\left(y_{0}\right)\right)} .
$$

The wave action flux is thus a constant and (3.20) is satisfied.

The singularities of the integrand in the expression (3.14) for $\Psi_{0}$ are

1. simple pole at $\sigma=0$ : steady solution,

2. branch point at $\sigma=\alpha U\left(y_{0}\right)$ : transient,

3. branch point at $\sigma=\alpha U(y)$ : transient.

Also, as $y \rightarrow y_{0}$, the branch points at $\sigma=\alpha U\left(y_{0}\right)$ and $\sigma=\alpha U(y)$ coalesce and saddle points at

$$
\sigma_{ \pm}=\alpha U\left(y_{0}\right) \pm \sqrt{y_{0}-y}\left(\alpha^{2} J R^{\prime}\left(y_{0}\right)\right)^{1 / 4} t^{-1 / 2}
$$

then occur.

Let us first consider the simple pole at $\sigma=0$. The dominant contribution from this pole lies behind the wavefront given by

$$
t=\int_{y}^{y_{0}} d \xi /\left|c_{g}\right|
$$

where the group velocity $c_{g}$ is found from (3.16) and (3.18) with $\sigma=0$. Therefore for

$$
t>\int_{y}^{y_{0}} \frac{\sqrt{J R^{\prime}(\xi)}}{\alpha(U(\xi))^{2}} d \xi
$$

the contribution of the pole is the steady solution

$$
\Psi_{0 s}=A e^{s \theta}
$$


where

$$
\begin{aligned}
A & =a\left(\frac{R^{\prime}\left(y_{0}\right)}{R^{\prime}(y)}\right)^{1 / 4} \sqrt{\frac{U\left(y_{0}\right)}{U(y)}} \\
\theta & =\int_{y}^{y_{0}} \sqrt{\frac{J R^{\prime}(\xi)}{U(\xi)}} d \xi \\
a & =\lim _{s \rightarrow 0} s F(s)
\end{aligned}
$$

(for $t<\int_{y}^{y_{0}} \sqrt{J R^{\prime}(\xi)} / \alpha(U(\xi))^{2} d \xi, \Psi_{0 s} \approx 0$ ).

The wave action flux can be found from (3.21) and it is

$$
c_{g} A \propto a^{2} \sqrt{J R^{\prime}\left(y_{0}\right)} / \alpha^{2} U\left(y_{0}\right) .
$$

The wave action flux of the steady solution is thus a constant and so the wave action equation (3.20) is satisfied.

As $y \rightarrow y_{0}$,

$$
\Psi_{0 s} \sim a \exp \left[-i \frac{\sqrt{J R^{\prime}\left(y_{0}\right)}}{U\left(y_{0}\right)}\left(y-y_{0}\right)\right],
$$

which is a wave propagating downwards. Near the critical layer around $y=0$, $U(y) \sim U^{\prime}(0) y$. Therefore as $y \rightarrow 0^{+}$,

$$
\Psi_{0 s} \sim a_{0} \exp \left(i \theta_{0}\right)
$$

where

$$
\begin{aligned}
A_{0} & =a\left(\frac{R^{\prime}\left(y_{0}\right)}{R^{\prime}(y)}\right)^{1 / 4} \sqrt{\frac{U^{\prime}(0)}{U^{\prime}\left(y_{0}\right)}} y^{1 / 2}, \\
\theta_{0} & =\frac{\sqrt{J R^{\prime}\left(y_{0}\right)} y_{0}}{U\left(y_{0}\right)}-\sqrt{J} \Omega-\frac{\sqrt{J R^{\prime}(0)}}{U^{\prime}(0)} \log y \\
\Omega & =\int_{0}^{\infty}\left(\frac{\sqrt{R^{\prime}(0)}}{U^{\prime}(0) \xi(\xi+1)}+\frac{\sqrt{R^{\prime}(\infty)}}{U(\infty)}-\frac{\sqrt{R^{\prime}(\xi)}}{U(\xi)}\right) d \xi .
\end{aligned}
$$

We see that near the critical layer, the derivatives of $\Psi_{0 s}$ are large, and that the viscous terms in the governing equations will be important there. In particular, the horizontal velocity $u_{0}$ is $\mathrm{O}\left(y^{-1 / 2}\right)$ and the vertical velocity $v_{0}$ is $\mathrm{O}\left(y^{1 / 2}\right)$ near the critical layer. As the critical layer is approached, the wave action density for the steady solution is $A \propto y^{-2}$. This result was found by Grimshaw [12] from the averaged equations for a slowly varying wave packet approaching a critical layer.

We shall now calculate the contribution from the branch point at $\sigma=\alpha U\left(y_{0}\right)$. The dominant contribution from this branch point lies behind the wavefront

$$
t=\int_{y}^{y_{0}} d \xi /\left|c_{g}\right|
$$


where $c_{g}$ is found from (3.16) and (3.18) with $\sigma=\alpha U\left(y_{0}\right)$. So for $t$ large and

$$
t>\int_{y}^{y_{0}} \frac{\sqrt{J R^{\prime}(\xi)} d \xi}{\alpha\left[U\left(y_{0}\right)-U(\xi)\right]^{2}}
$$

the contribution from the branch point at $\sigma=\alpha U\left(y_{0}\right)$ is

$$
\Psi_{01} \sim\left(\frac{R^{\prime}\left(y_{0}\right)}{R^{\prime}(y)}\right)^{1 / 4} \sqrt{\alpha\left(U\left(y_{0}\right)-U(y)\right)} e^{-i \alpha U\left(y_{0}\right) t} F\left(-i \alpha U\left(y_{0}\right)\right) I
$$

where

$$
\begin{aligned}
& I=\frac{1}{2 \pi \sqrt{t}} \int_{-\infty}^{\infty} u^{-1 / 2} e^{-i u-i \chi} d u \\
& \chi=\int_{y}^{y_{0}} \frac{\alpha t \sqrt{J R^{\prime}(\xi)} d \xi}{u+\alpha t\left(U\left(y_{0}\right)-U(\xi)\right)}
\end{aligned}
$$

Expanding $\chi$ to second order in $t$ gives

$$
I \sim I_{0} t^{-1 / 2} \exp \left[-i\left(\chi_{0}+\frac{\mu_{0} \chi_{1}}{\alpha t}+\cdots\right)\right]
$$

where

$$
\begin{aligned}
I_{0}= & \frac{1}{2 \pi} \int_{-\infty}^{\infty} u^{-1 / 2+i \mu_{0}} e^{-i u} d u \\
\chi_{0}= & \mu_{0} \log \left[\alpha t U^{\prime}\left(y_{0}\right)\left(y_{0}-y\right)\right] \\
& +\int_{y}^{y_{0}}\left[\frac{\sqrt{J R^{\prime}(\xi)}}{U\left(y_{0}\right)-U(\xi)}-\frac{\sqrt{J R^{\prime}\left(y_{0}\right)}}{U^{\prime}\left(y_{0}\right)\left(y_{0}-\xi\right)}\right] d \xi \\
\chi_{1}= & \mu_{0} / U^{\prime}\left(y_{0}\right)\left(y_{0}-y\right)-\int_{y}^{y_{0}}\left[\frac{\sqrt{J R^{\prime}(\xi)}}{\left(U\left(y_{0}\right)-U(\xi)\right)^{2}}-\frac{\sqrt{J R^{\prime}\left(y_{0}\right)}}{\left(U^{\prime}\left(y_{0}\right)\right)^{2}\left(y_{0}-\xi\right)^{2}}\right] d \xi \\
\mu_{0}= & \frac{\sqrt{J R^{\prime}\left(y_{0}\right)}}{U^{\prime}\left(y_{0}\right)}
\end{aligned}
$$

Since $J \gg 1$, the integral $I_{0}$ may be evaluated by the method of steepest descent. This yields

$$
I_{0} \sim \frac{1}{\sqrt{2 \pi}} \mu_{0}^{i \mu_{0}} e^{-i \mu_{0}-i \pi / 4} .
$$

The contribution due to the branch point at $\sigma=\alpha U\left(y_{0}\right)$ is therefore

$$
\Psi_{01}=A_{1} \exp \left(i \theta_{1}\right)
$$

where

$$
\begin{aligned}
\theta_{1}= & \mu_{0} \log \mu_{0}-\mu_{0}-\frac{\pi}{4}-\alpha U\left(y_{0}\right) t \\
& -\mu_{0} \log \left[\alpha t U^{\prime}\left(y_{0}\right)\left(y_{0}-y\right)\right]-\int_{y}^{y_{0}}\left[\frac{\sqrt{J R^{\prime}(\xi)}}{U\left(y_{0}\right)-U(\xi)}-\frac{\sqrt{J R^{\prime}\left(y_{0}\right)}}{U^{\prime}\left(y_{0}\right)\left(y_{0}-\xi\right)}\right] d \xi \\
& +\frac{\mu_{0}}{\alpha t} \int_{y}^{y_{0}}\left[\frac{\sqrt{J R^{\prime}(\xi)}}{\left(U\left(y_{0}\right)-U(\xi)\right)^{2}}-\frac{\sqrt{J R^{\prime}\left(y_{0}\right)}}{\left(U^{\prime}\left(y_{0}\right)\right)^{2}\left(y_{0}-\xi\right)^{2}}\right] d \xi
\end{aligned}
$$


and

$$
A_{1}=\frac{t^{-1 / 2}}{\sqrt{2 \pi}}\left(\frac{R^{\prime}\left(y_{0}\right)}{R^{\prime}(y)}\right)^{1 / 4} \sqrt{\alpha\left(U\left(y_{0}\right)-U(y)\right)} F\left(-i \alpha U\left(y_{0}\right)\right) .
$$

From the phase function (3.36), we can evaluate the frequency $\omega_{1}$ and the wavenumber $m_{1}$ of the wave as

$$
\begin{aligned}
& \omega_{1}=\alpha U\left(y_{0}\right)+\mu_{0} / t+\mathrm{O}\left(1 / t^{2}\right) \\
& m_{1}=\frac{\sqrt{J R^{\prime}(y)}}{U\left(y_{0}\right)-U(y)}-\frac{\mu_{0}}{\alpha t} \frac{\sqrt{J R^{\prime}(y)}}{\left(U\left(y_{0}\right)-U(y)\right)^{2}}+\mathrm{O}\left(1 / t^{2}\right) .
\end{aligned}
$$

This wave thus satisfies the dispersion relation (3.17) for internal gravity waves, as required. The wave action flux is

$$
c_{g} A_{1} \propto \sqrt{R}^{\prime}\left(y_{0}\right)\left|F\left(-i \alpha U\left(y_{0}\right)\right)\right|^{2} t^{-1}+\mathrm{O}\left(t^{-2}\right) .
$$

The wave action density $A_{1} \propto t^{-1}$ and the wave action equation (3.20) is satisfied to $\mathrm{O}\left(t^{-2}\right)$.

Near the critical layer, the wavenumber $m_{1}$ of the wave (3.35) is constant, the group velocity is constant and the wave has no singular behaviour there. This wave decays uniformly as $t \rightarrow \infty$ and makes a negligible contribution to the development of the critical layer.

We shall finally consider the contribution from the branch point at $\sigma=\alpha U(y)$. The dominant contribution from this branch point lies in the region

$$
t>\int_{y}^{y_{0}} d \xi /\left|c_{g}\right|
$$

where the group velocity is found from (3.16) and (3.18) on setting $\sigma=\alpha U(y)$.

In a similar manner as used above for the branch point at $\sigma=\alpha U\left(y_{0}\right)$, it may be shown that the contribution from the branch point at $\sigma=\alpha U(y)$ is

$$
\Psi_{02}=A_{2} \exp \left(i \theta_{2}\right)
$$

for $t$ large and

$$
t>\int_{y}^{y_{0}} \frac{\sqrt{J R^{\prime}(\xi)}}{\alpha[U(y)-U(\xi)]^{2}} d \xi
$$

where

$$
\begin{aligned}
& \theta_{2}=-\mu \log \mu-\mu+\pi / 4-\alpha U(y) t+\mu \log \left[\alpha t\left(y_{0}-y\right) U^{\prime}(y)\right] \\
&-\int_{y}^{y_{0}}\left[\frac{\sqrt{J R^{\prime}(\xi)}}{U(y)-U(\xi)}-\frac{\sqrt{J R^{\prime}(y)}}{U^{\prime}(y)(y-\xi)}\right] d \xi \\
& A_{2}=\frac{\mu}{\sqrt{2 \pi}}\left(\frac{R^{\prime}\left(y_{0}\right)}{R^{\prime}(y)}\right)^{1 / 4}\left[\alpha\left(U\left(y_{0}\right)-U(y)\right)\right]^{-1 / 2} F(-i \alpha U(y)) t^{-3 / 2}
\end{aligned}
$$


and

$$
\mu=\sqrt{J R^{\prime}(y)} / U^{\prime}(y) .
$$

Again, it may be shown that this wave satisfies the dispersion relation (3.17) for internal gravity waves and the wave action equation (3.20). This transient decays as $t^{-3 / 2}$, except near $y=0$, where it grows as $y^{-1}$ due to the pole of $F(s)$ at $s=0$. This transient is associated with the impulsive start of the wave source. The group velocity for this transient is

$$
c_{g}=-\frac{\sqrt{J R^{\prime}(y)}}{\alpha t^{2}\left(U^{\prime}(y)\right)^{2}}
$$

and the wave action flux is

$$
c_{g} \AA^{\propto} \frac{\sqrt{R^{\prime}(y)}|F(-i \alpha U(y))|^{2}}{U^{\prime}(y)\left(U\left(y_{0}\right)-U(y)\right) t^{2}} .
$$

The group velocity in the critical layer is small $\left(\propto t^{-2}\right)$. The wave action flux in the critical layer is large $\left(\propto y^{-2} t^{-2}\right)$ due to the pole of $F(s)$ at $s=0$. Wave action is piling up in the critical layer since, in this linear inviscid theory, the critical layer acts as a barrier to the propagation of wave action, which is continually being added by the wave source at $y_{0}$. As $y \rightarrow 0$, the pole at $\sigma=0$ and the branch point at $\sigma=\alpha U(y)$ coalesce. The critical layer will be examined in detail in the next section.

The solution outside the critical layer for large times thus consists of three slowly varying waves, each of which satisfy the dispersion relation for internal gravity waves. One of these waves is the steady state and the other two are transients. The transient $\Psi_{01}$ (due to the branch point at $\sigma=\alpha U\left(y_{0}\right)$ ) decays uniformly while the transient $\Psi_{02}$ (due to the branch point at $\sigma=\alpha U(y)$ ) becomes negligible everywhere except near the critical layer.

\section{Linear, viscous critical layer}

Near the critical layer, $y \approx 0$, the derivatives of $\bar{\Psi}$ become large and the viscous terms in equation (3.6) become important. The behaviour in the critical layer is further complicated by the coalescence of the pole at $\sigma=0$ and the branch point at $\sigma=\alpha U(y)$ in the integrand of the integral representation of the outer solution (3.14). To examine the critical layer, we define critical layer variables by

$$
Y=y(\mathrm{Re})^{1 / 3}, \quad s^{*}=s(\mathrm{Re})^{1 / 3}
$$

and a critical layer expansion by

$$
\bar{\Psi}_{\text {inner }}=\bar{\phi}_{0}\left(Y, s^{*}\right)+(\operatorname{Re})^{-1} \bar{\phi}_{1}\left(Y, s^{*}\right)+\cdots .
$$



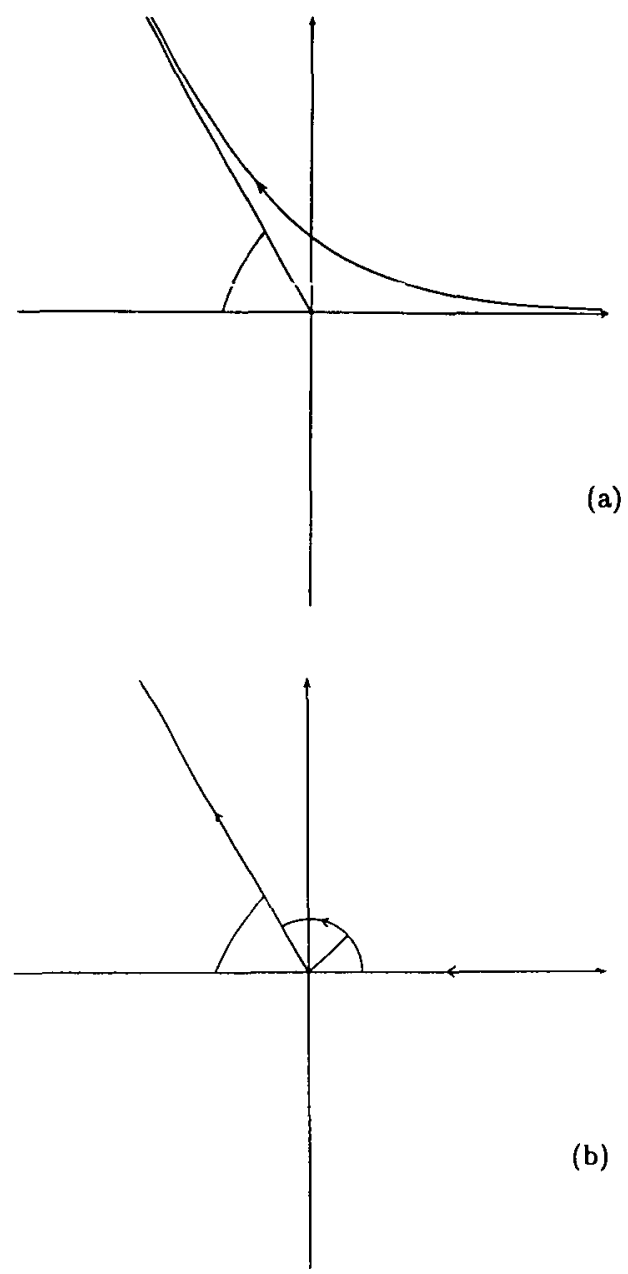

Figure 2. (a) Contour of integration $C_{3}$ for critical layer solution.

(b) Deformed contour of integration for critical layer solution.

From equation (3.6), we obtain the first order critical layer equation

$$
\begin{aligned}
& \operatorname{Pr}^{-1} \partial^{6} \bar{\phi}_{0} / \partial Y^{6}-\left(1+\operatorname{Pr}^{-1}\right)\left(s^{*}+i \alpha U_{0}^{\prime} Y\right) \partial^{4} \bar{\phi}_{0} / \partial Y^{4} \\
& \quad-2 i \alpha \operatorname{Pr}^{-1} U_{0}^{\prime} \partial^{3} \bar{\phi}_{0} / \partial Y^{3}+\left(s^{*}+i \alpha U_{0}^{\prime} Y\right)^{2} \partial^{2} \bar{\phi}_{0} / \partial Y^{2}-\alpha^{2} J R_{0}^{\prime} \bar{\phi}_{0}=0,
\end{aligned}
$$

where

$$
U_{0}^{\prime}=U^{\prime}(0), \quad R_{0}^{\prime}=R^{\prime}(0)
$$

and the Prandtl number $\mathrm{Pr}$ is

$$
\operatorname{Pr}=\operatorname{RePe}
$$


Baldwin and Roberts [1] considered the steady solution in the viscous critical layer. Their critical layer equation is similar to (4.3) with $s^{*}=0$ (as the steady solution corresponds to a pole at $s^{*}=0$ for the Laplace transform solution). The solution of (4.3) with the correct behaviour to match with the outer solution as $Y \rightarrow \infty$ is

$$
\bar{\phi}_{0}=A\left(s^{*}\right) \int_{C_{3}} \exp \left[-\frac{\left(s^{*}+i \alpha U_{0}^{\prime} Y\right) \xi}{\alpha^{2 / 3} U_{0}^{\prime 2 / 3}}-\frac{1}{3} \xi^{3}\right] \frac{f(\xi)}{\xi^{k}} d \xi,
$$

where

$$
\begin{gathered}
f(\xi)={ }_{1} F_{1}\left(\frac{1}{6}+\frac{1}{3} i \bar{\mu}, 1+\frac{2}{3} i \bar{\mu} ; \frac{1}{3}\left(1-\mathrm{Pr}^{-1}\right) \xi^{3}\right), \\
k=\frac{3}{2}-i \bar{\mu}, \quad \bar{\mu}=\sqrt{J R_{0}^{\prime}} / U_{0}^{\prime} .
\end{gathered}
$$

The contour $C_{3}$ starts at $\infty e^{i 0}$ and terminates at $\infty e^{2 \pi z / 3}$. This contour is sketched in Figure 2(a). ${ }_{1} F_{1}$ is the confluent hypergeometric function. We shall now take the particular value $\operatorname{Pr}=\infty$ for the Prandtl number (or to be more precise, we shall take $\mathrm{Pe} \gg \mathrm{Re}$ ). In the remainder of the present work, we shall be concerned with the velocity in the critical layer and this choice of the Prandtl number has no significant effect on the behaviour of the critical layer velocity.

The function $A\left(s^{*}\right)$ is obtained by matching with the outer solution. As $s^{*}+i \alpha U_{0}^{\prime} Y \rightarrow \infty \exp (i \pi / 2)$, the method of steepest descent gives

$$
\bar{\phi}_{0} \sim A\left(s^{*}\right) \frac{2 \pi}{\Gamma(k)} e^{-\pi \bar{\mu}}\left(\frac{s^{*}+i \alpha U_{0}^{\prime} Y}{\alpha^{2 / 3} U_{0}^{\prime 2 / 3}}\right)^{1 / 2-i \bar{\mu}}
$$

From the outer solution given by (3.9) and (3.10), it can be seen that as $y \rightarrow 0$,

$$
\begin{aligned}
\bar{\Psi}_{0} \sim & \left(R^{\prime}\left(y_{0}\right) / R_{0}^{\prime}\right)^{1 / 4}\left(s+i \alpha U\left(y_{0}\right)\right)^{-1 / 2}\left(s+i \alpha U_{0}^{\prime} y_{0}\right)^{i \bar{\mu}}\left(s+i \alpha U_{0}^{\prime} y\right)^{1 / 2-i \bar{\mu}} \\
& \times \frac{a}{s} \exp \left[-\int_{y}^{y_{0}}\left(\frac{\alpha \sqrt{J R^{\prime}(\xi)}}{s+i \alpha U(\xi)}-\frac{\alpha \sqrt{J R_{0}^{\prime}}}{s+i \alpha U_{0}^{\prime} \xi}\right) d \xi\right]
\end{aligned}
$$

Thus

$$
\begin{aligned}
A\left(s^{*}\right)= & \frac{\Gamma(k)}{2 \pi}\left(\frac{R^{\prime}\left(y_{0}\right)}{R_{0}^{\prime}}\right)^{1 / 4}(\mathrm{Re})^{1 / 6+i \bar{\mu} / 3} \frac{a}{s^{*}}\left(\alpha U\left(y_{0}\right)\right)^{-1 / 2} \\
& \times \exp (-\pi i / 4+\pi \mu / 2)\left(\alpha U_{0}^{\prime} y_{0}\right)^{i \bar{\mu}}\left(\alpha U_{0}^{\prime}\right)^{1 / 3-2 i \bar{\mu} / 3} \\
& \times \exp \left[i \int_{0}^{y_{0}}\left(\frac{\sqrt{J R^{\prime}(\xi)}}{U(\xi)}-\frac{\sqrt{J R_{0}^{\prime}}}{U_{0}^{\prime} \xi}\right) d \xi\right] .
\end{aligned}
$$

We see that the viscous critical layer smooths out the discontinuities in the outer solution. 
From the expression (4.6) for $\bar{\phi}_{0}$, we find that as $s^{*}+i \alpha U_{0}^{\prime} Y \rightarrow \infty \exp (-i \pi / 2)$,

$$
\bar{\phi}_{0} \sim A\left(s^{*}\right) \frac{2 \pi}{\Gamma(k)} e^{-2 \pi \bar{\mu}}\left(\frac{s^{*}+i \alpha U_{0}^{\prime} Y}{\alpha^{2 / 3} U_{0}^{\prime 2 / 3}}\right)^{1 / 2-i \bar{\mu}}
$$

This shows the exponential damping of the wave as it passes through the critical layer. Below the critical layer, the inner solution is matched with an outer solution, which has the same form as the dominant terms in the outer solution above the critical layer, except that it is multiplied by a factor $\exp (-\pi \tilde{\mu})$. This result was found by Booker and Bretherton [2] in their investigation of the (linear) critical layer.

Hazel [14] considered the steady-state viscous critical layer for the case when the Prandtl number is finite and the Richardson number is $\mathrm{O}(1)$. The criticallayer equation found is the same as (4.3) with $s^{*}=0$, as this corresponds to the steady-state solution. This equation was integrated numerically, and the exponential damping of the wave across the critical layer was found.

In the critical layer, the nonlinear terms in the full evolution equations (2.5) and (2.6) are $O\left(\varepsilon^{2}(\mathrm{Re})^{2 / 3}\right)$ and the viscous terms are $O\left(\varepsilon(\operatorname{Re})^{1 / 6}\right)$. Therefore, for the present analysis to be valid, we require

$$
\varepsilon \ll(\operatorname{Re})^{-1 / 2} .
$$

The contour of integration $C_{3}$ for the expression (4.6) for $\bar{\phi}_{0}$ can be deformed to the real axis from $\infty$ to $\delta$, the line $\xi=r \exp [2 \pi i / 3], r \in(\delta, \infty)$, and an arc of a small circle of radius $\delta$ around the origin joining these two lines. This deformed contour is sketched in Figure 2(b). The integral along the line $\xi=r \exp [2 \pi i / 3]$ is negligible as it is multiplied by a factor $\exp [-2 \pi \tilde{\mu} / 3]$. Therefore, $\phi_{0}$ is given approximately by

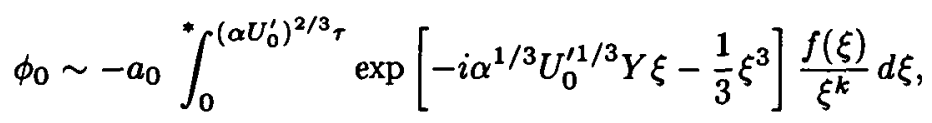

where

$$
\begin{aligned}
& \tau=t(\mathrm{Re})^{-1 / 3} \\
& a_{0}= \lim _{s^{*} \rightarrow 0} s^{*} A\left(s^{*}\right) \\
&= \frac{a \Gamma(k)}{2 \pi}\left(\frac{R^{\prime}\left(y_{0}\right)}{R_{0}^{\prime}}\right)^{1 / 4} \operatorname{Re}^{1 / 6+i \bar{\mu} / 3} e^{-\pi i / 4+\pi \bar{\mu} / 2}\left(\alpha U\left(y_{0}\right)\right)^{-1 / 2} \\
& \times\left(\alpha U_{0}^{\prime}\right)^{1 / 3-2 i \bar{\mu} / 3}\left(\alpha U_{0}^{\prime} y_{0}\right)^{i \bar{\mu}} \exp \left[i \int_{0}^{y_{0}}\left(\frac{\sqrt{J R^{\prime}(\xi)}}{U(\xi)}-\frac{\sqrt{J R_{0}^{\prime}}}{U_{0}^{\prime} \xi}\right) d \xi\right]
\end{aligned}
$$


and ${ }^{*}$ denotes that the finite part of the integral is to be taken. If we take $\tau \gg 1$ and then let $Y \rightarrow \infty$, we find

$$
\begin{aligned}
\phi_{0} \sim & \frac{2 \pi a_{0}}{\Gamma(k)} e^{i \pi / 4-\pi \bar{\mu} / 2}\left(\alpha^{1 / 3} U_{0}^{\prime 1 / 3} Y\right)^{1 / 2-i \bar{\mu}} \\
& -i a_{0}\left(\alpha U_{0}^{\prime}\right)^{-4 / 3+2 i \bar{\mu} / 3} Y^{-1} \tau^{i \bar{\mu}-3 / 2} e^{-i \alpha U_{0}^{\prime} Y \tau} .
\end{aligned}
$$

The first term in this expression matches with the steady solution (3.28) in the outer region and the second term matches with the transient (3.41) in the outer region.

For large time, the solution in the critical layer is essentially the steady solution. This steady solution is

$$
\phi_{0 s}=-a_{0} \int_{0}^{*} \exp \left[-i \alpha^{1 / 3} U_{0}^{1 / 3} Y \xi-\frac{1}{3} \xi^{3}\right] \frac{f(\xi)}{\xi^{k}} d \xi
$$

In the next section, we shall need the asymptotic form of this integral as $Y \rightarrow \infty$ to second order. The method of steepest descent gives

$$
\begin{aligned}
\phi_{0 s} \sim & \frac{2 \pi}{\Gamma(k)} a_{0} e^{\pi i / 4-\pi \bar{\mu} / 2}\left(\alpha U_{0}^{\prime}\right)^{1 / 6-i \bar{\mu} / 3} Y^{1 / 2-i \bar{\mu}} \\
& +\frac{\pi a_{0}}{3 \Gamma(k-3)} e^{3 \pi i / 4-\pi \bar{\mu} / 2}\left(\alpha U_{0}^{\prime}\right)^{-5 / 6-i \bar{\mu} / 3} Y^{-5 / 2-i \bar{\mu}}+\cdots .
\end{aligned}
$$

The integral in the expression (4.16) for $\phi_{0 s}$ has been evaluated numerically. Let us set $u_{0 s}^{i}=\phi_{0 s Y}^{i}, u_{0 s}^{i}$ being the leading order steady wave horizontal velocity in the critical layer. In Figures 3(a) and 3(b), $\left|u_{0 s}^{i}\right| /\left|a_{0}\right|$ is plotted as a function of $Y$ for $\bar{\mu}=25$ and $\bar{\mu}=50$ respectively. The outer steady horizontal velocity in the limit $y \rightarrow 0$, given by (3.28) and (3.29), has also been plotted in these figures as a dashed curve. The attenuation of the horizontal velocity due to the action of viscosity as the critical level is approached can be seen.

\section{Wave-induced mean flow}

The perturbation produced by the forcing will generate a mean flow due to the nonlinear terms in the governing equations (2.5) and (2.6). To find this mean flow acceleration, we expand $\psi$ and $T$ in a small amplitude expansion as

$$
\psi=\varepsilon \psi_{1}+\varepsilon^{2} \psi_{2}+\cdots, \quad T=\varepsilon T_{1}+\varepsilon^{2} T_{2}+\cdots
$$

where $\psi_{1}$ and $T_{1}$ are the linear solutions found in Sections 3 and 4 . At the first order, no mean flow is produced as $\psi_{1}$ and $T_{1}$ are periodic in $x$. Substituting these series in the governing equations (2.5) and (2.6), we find at $O\left(\varepsilon^{2}\right)$ the equations

$$
\left(\frac{\partial}{\partial t}+U(y) \frac{\partial}{\partial x}\right) \nabla^{2} \psi_{2}-U^{\prime \prime}(y) \psi_{2 x}+J T_{2 x}-(\mathrm{Re})^{-1} \nabla^{4} \psi_{2}=\partial\left(\psi_{1}, \nabla^{2} \psi_{1}\right) / \partial(x, y)
$$



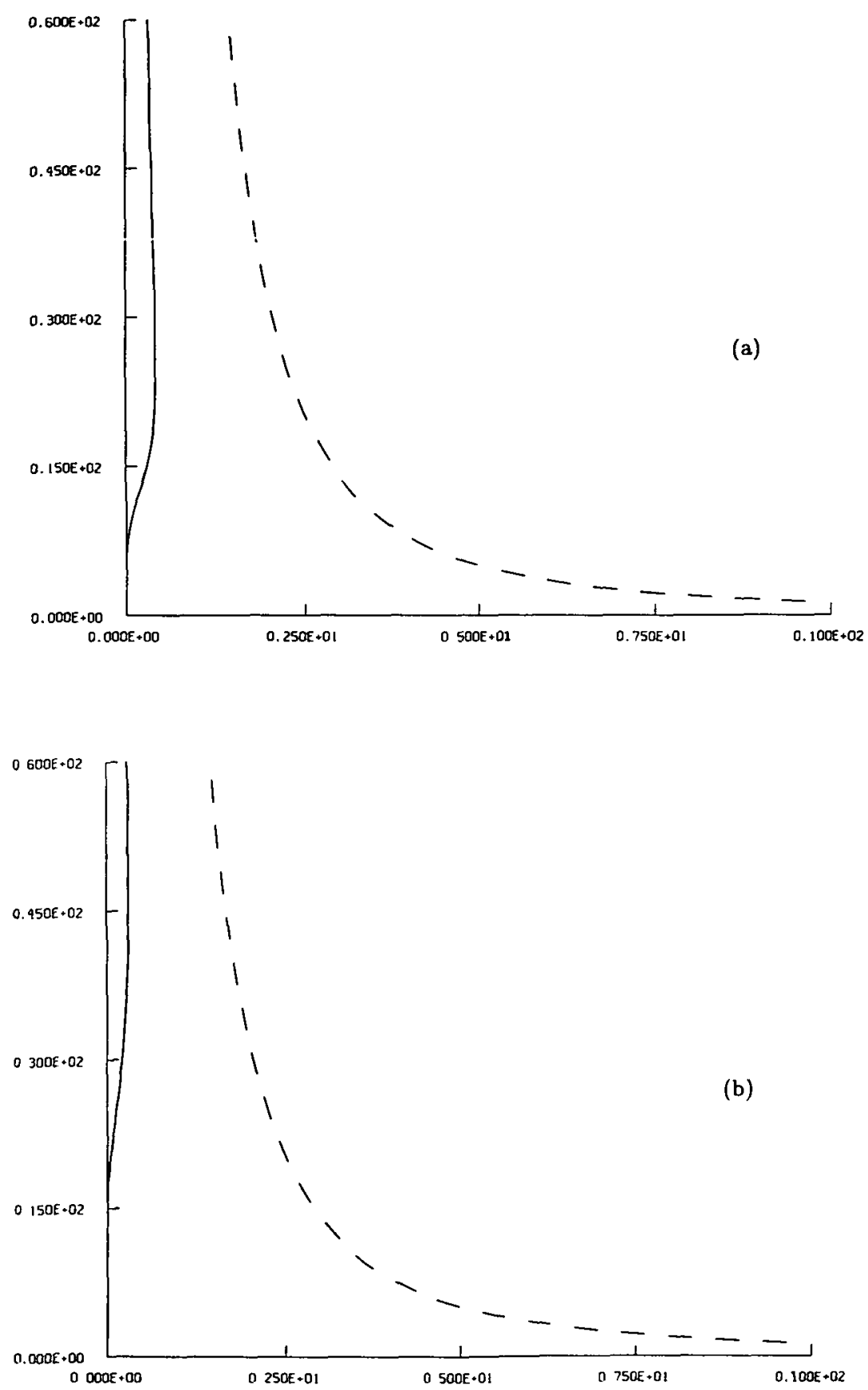

Figure 3. Modulus of critical layer steady wave horizontal velocity $u_{0 s}^{i}$

(a) $\bar{\mu}=25$ : - modulus of linear, inviscid velocity in critical layer: - - -

(b) $\bar{\mu}=50$ : - modulus of linear, inviscid velocity in critical layer: - - - 


$$
\left(\frac{\partial}{\partial t}+U(y) \frac{\partial}{\partial x}\right) T_{2}+R^{\prime}(y) \psi_{2 x}=\partial\left(\psi_{1}, T_{1}\right) / \partial(x, y),
$$

on taking $\operatorname{Pr}=\infty$. The first order solution $\left(\psi_{1}, T_{1}\right)$ is periodic in $x$. To find the behaviour of the mean flow at second order, the equations (5.2) and (5.3) are averaged with respect to $x$. This yields

$$
\begin{array}{r}
\left(\frac{\partial}{\partial t}+U(y) \frac{\partial}{\partial x}\right) \nabla^{2} \bar{\psi}_{2}-U^{\prime \prime}(y) \bar{\psi}_{2 x}+J \bar{T}_{2 x}-(\operatorname{Re})^{-1} \nabla^{4} \bar{\psi}_{2}=\overline{\partial\left(\psi_{1}, \nabla^{2} \psi_{1}\right) / \partial(x, y)} \\
\left(\frac{\partial}{\partial t}+U(y) \frac{\partial}{\partial x}\right) \bar{T}_{2}+R^{\prime}(y) \bar{\psi}_{2 x}=\overline{\partial\left(\psi_{1}, T_{1}\right) / \partial(x, y)},
\end{array}
$$

where the overbar denotes averages. The forcing terms on the right hand side of the equations are independent of $x$, so the mean quantities $\bar{\psi}_{2}$ and $\bar{T}_{2}$ are also independent of $x$. On integrating equation (5.4) twice, we find that the mean second order stream function satisfies

$$
\partial^{2} \bar{\psi}_{2} / \partial t \partial y-(\mathrm{Re})^{-1} \partial^{3} \bar{\psi}_{2} / \partial y^{3}=\frac{\partial}{\partial y}\left(\overline{\psi_{1 x} \psi_{1 y}}\right) .
$$

This equation is a forced heat equation for the wave-induced mean flow $\bar{u}_{2}=\bar{\psi}_{2 y}$, with the forcing function being the $y$-derivative of the Reynolds stress

$$
\Sigma=\overline{\psi_{1 x} \psi_{1 y}} \text {. }
$$

The solution of this equation is

$$
\bar{u}_{2}=\int_{0}^{t} \int_{-\infty}^{\infty} \frac{\sqrt{\mathrm{Re}}}{\sqrt{4 \pi\left(t-t^{\prime}\right)}} \exp \left[-\frac{(y-\xi)^{2} \mathrm{Re}}{4\left(t-t^{\prime}\right)}\right] \frac{\partial}{\partial \xi}\left(\Sigma\left(t^{\prime}, \xi\right)\right) d \xi d t^{\prime} .
$$

For large times, the major contribution to $\bar{u}_{2}$ comes from the steady part of the Reynolds stress, which will be denoted by $\Sigma_{s}$. This stress consists of two components: the contribution from the outer region and the contribution from the critical layer. The Reynolds stress in the outer region is, from (3.1) and (3.24),

$$
\Sigma_{s}^{0}=\overline{\psi_{s x}^{0} \psi_{s y}^{0}}=-2 \alpha a^{2} \frac{\sqrt{J R^{\prime}\left(y_{0}\right)}}{U\left(y_{0}\right)} .
$$

Here $\psi_{s}^{0}$ refers to the steady solution in the outer region found in Section 3. From (3.26), it can be seen that this Reynolds stress in the outer region is proportional to the wave action flux, $c_{g} A$, for the outer steady solution.

The Reynolds stress behind the wavefront of the outer steady solution is thus constant and makes no contribution to the induced mean flow. However, there is a contribution to the Reynolds stress due to the front of the outer steady solution, as at the front, $\Sigma_{y}$ is proportional to a $\delta$-function. Grimshaw [13] showed that this contribution is proportional to the wave action density $A_{s}$ of the outer steady solution and is $\mathrm{O}\left(\varepsilon^{2}\right)$. It will be shown below that the critical 
layer makes a contribution $\mathrm{O}\left(\varepsilon^{2} \sqrt{\mathrm{Re}} \sqrt{t}\right)$ to the induced mean flow. The mean flow (for large time) is hence due mainly to the critical layer Reynolds stress and is approximately

$$
\bar{u}_{2}=\int_{0}^{\tau} \int_{-\infty}^{\infty} \frac{\mathrm{Re}}{\sqrt{4 \pi\left(\tau-t^{\prime}\right)}} \exp \left[-\frac{(Y-\xi)^{2}}{4\left(\tau-t^{\prime}\right)}\right] \frac{\partial}{\partial \xi} \Sigma_{s}^{i}(\xi) d \xi d t^{\prime}
$$

where

$$
\begin{gathered}
Y=(\operatorname{Re})^{1 / 3} y, \quad \tau=(\operatorname{Re})^{-1 / 3} t, \\
\Sigma_{s}^{i}=\overline{\psi_{s 1 x}^{i}(x, Y) \psi_{s 1 Y}^{i}(x, Y) .}
\end{gathered}
$$

Here $\Sigma_{s}^{i}$ refers to the Reynolds stress in the critical layer due to the steady solution and $\psi_{s 1}^{i}$ is the leading order steady solution in the critical layer found in Section 4.

The behaviour of $\bar{u}_{2}$ can be found by asymptotic evaluation of the integral in (5.10). For small $\tau$, it can be found from (5.10) that

$$
\bar{u}_{2} \sim(\mathrm{Re}) \tau \frac{\partial}{\partial Y} \Sigma_{s}^{i}
$$

Initially, before the effects of viscosity become important, the induced horizontal velocity grows linearly with time. As time increases, the vorticity produced by the critical layer will diffuse outwards and the mean flow associated with this vorticity will eventually dominate over the induced mean velocity (5.12).

As $Y \rightarrow \infty$, the mean flow is always dominated by non-viscous effects and (5.12) is the asymptotic form of (5.10) as $Y \rightarrow \infty$. The Reynolds stress for large $Y$ can be evaluated on using (4.14) and (4.15) and asymptotically evaluating the gamma function as $|k|$ is large. We find that

$$
\begin{aligned}
\Sigma_{s}^{i} \sim & -2 \alpha a^{2}(\mathrm{Re})^{-1 / 3} \sqrt{J R^{\prime}\left(y_{0}\right)} / U\left(y_{0}\right) \\
& +\frac{2}{3} a^{2}(\mathrm{Re})^{-1 / 3} \overline{\mu^{4}} e^{-3} \sqrt{R^{\prime}\left(y_{0}\right) / R_{0}^{\prime}}\left(U\left(y_{0}\right)\right)^{-1} Y^{-3} .
\end{aligned}
$$

So for $\tau$ fixed and $Y \rightarrow \infty$,

$$
\bar{u}_{2} \sim-2(\operatorname{Re})^{2 / 3} a^{2} \bar{\mu}^{4} e^{-3} \sqrt{R^{\prime}\left(y_{0}\right) / R_{0}^{\prime}}\left(U\left(y_{0}\right)\right)^{-1} \tau Y^{-4} .
$$

As $Y \rightarrow \infty$, the induced mean flow decays as $Y^{-4}$ and grows linearly with $\tau$. The behaviour as $Y \rightarrow-\infty$ for finite $\tau$ is the same, except that the right hand side of $(5.14)$ is multiplied by the factor $-e^{-2 \pi \bar{\mu}}$. The induced mean velocity (5.14) is $\mathrm{O}\left(\mathrm{Re}^{-1}\right)$ and so is due to the second order outer solution, which is $\mathrm{O}\left(\mathrm{Re}^{-1}\right)$. This induced mean velocity is thus forced by the outer flow.

Grimshaw [12] showed that for a slowly varying wave approaching a critical layer, the induced mean flow is given by

$$
\rho_{0} \bar{u}_{t}=\alpha\left(\mathcal{A}_{t}+\lambda \nu^{2} A\right),
$$


where

$$
\lambda \propto(\mathrm{Re})^{-1}
$$

and $\nu$ is the wavenumber. Therefore as $t \rightarrow \infty$,

$$
\bar{u} \sim \frac{\alpha}{\rho_{0}} \lambda \nu^{2} A t .
$$

Using (3.21) and (3.29), we then find that as $t \rightarrow \infty$

$$
\begin{aligned}
\bar{u} \propto & -\alpha(\mathrm{Re})^{-1} t y^{-4} \\
& =-\alpha(\mathrm{Re})^{2 / 3} \tau Y^{-4} .
\end{aligned}
$$

This expression agrees with the induced mean flow (5.14) far from the critical layer.

To find the mean velocity induced by viscous effects in the critical layer, the expression (5.10) for $\bar{u}_{2}$ is asymptotically evaluated for $Y$ fixed and $\tau$ large. For $Y$ fixed and $\tau \rightarrow \infty$, it can be found by integrating (5.10) by parts that

$$
\bar{u}_{2} \sim \frac{\operatorname{Re} \sqrt{\tau}}{\sqrt{\pi}}\left[\Sigma_{s}^{2}(\infty)-\Sigma_{s}^{2}(-\infty)\right]
$$

The Reynolds stress at $Y=-\infty$ is exponentially small and the Reynolds stress at $Y=\infty$ is given by (5.13). Hence for $Y$ fixed and $\tau \rightarrow \infty$,

$$
\bar{u}_{2} \sim-\frac{2 \alpha a^{2}}{\sqrt{\pi}}(\mathrm{Re})^{2 / 3} \sqrt{\tau} \sqrt{J R^{\prime}\left(y_{0}\right)} / U\left(y_{0}\right) .
$$

The mean flow induced by the action of viscosity in the critical layer is negative and grows as $\sqrt{\tau}$. The expansion (5.1) then remains valid until $\tau=\mathrm{O}\left(\operatorname{Re}^{-1} \varepsilon^{-2}\right)$ (i.e. $\left.t=\mathrm{O}\left(\mathrm{Re}^{-2 / 3} \varepsilon^{-2}\right)\right)$. For times greater than this, the effect of the nonlinear, convective terms in the governing equations (2.5) and (2.6) must be considered in the critical layer. Viscosity acts to reduce the rate of temporal increase of the induced mean flow and hence delay the time at which nonlinear effects become important. However, viscosity cannot fully arrest the growth of the horizontal velocity and stop nonlinear effects from becoming important. The critical layer acts as a source of vorticity, which diffuses into the outer flow. The net result after a long time is that the outer flow has a term of $O\left(\varepsilon^{2}(\operatorname{Re})^{2 / 3} \sqrt{\tau}\right)\left(=O\left(\varepsilon^{2} \sqrt{\operatorname{Re}} \sqrt{t}\right)\right)$ subtracted from it.

We see from (5.20) that the acceleration of the mean flow has moved the critical level towards the incoming wave. Grimshaw [13] showed that for a slowly varying wave in a fluid where nonlinearity dominates, the mean flow induced by the passage of the wavefront is given by

$$
\bar{u}=\alpha A,
$$

Using (3.21), this becomes

$$
\bar{u}=-\mathcal{E} / U(y)
$$


as we are considering the steady solution. The induced mean flow in an inviscid fluid is thus negative for $y>0$ for the shear profiles $U(y)$ under consideration. So both nonlinearity and viscosity dominated critical layers result in the critical level being moved towards the incoming wave. The negative sign of the induced mean flow near the critical level when nonlinearity dominates over viscosity was also found in the numerical work of Dunkerton and Fritts [9] and Fritts and Dunkerton [10j]. In their work, $\varepsilon \sqrt{R e}$ was $O\left(10^{3}\right)$ to $O\left(10^{4}\right)$, when the Reynolds number is based on molecular viscosity. Even if the Reynolds number is based on eddy viscosity, $\varepsilon \sqrt{\mathrm{Re}}$ was still $\mathrm{O}\left(10^{2}\right)$ to $\mathrm{O}\left(10^{3}\right)$.

Brown, Rosen and Maslowe [3] discussed the interaction of an internal gravity wave with a critical layer when the undisturbed velocity profile is $U(y)=\tanh y$. The equations used were similar to (2.5) and (2.6), except that the Richardson number was taken to be $\frac{1}{4}$ and the Prandtl number 1 , as in this case, the equations have an exact solution. Furthermore, the critical layer solution was developed for a steady outer solution. The expressions they found for the induced mean flow were similar to (5.10) and (5.14). The major difference was that for $\tau$ fixed and $|Y| \rightarrow \infty$, they found $\bar{u}_{2}=\mathrm{O}\left(\tau|Y|^{-2}\right)$, rather than $\bar{u}_{2}=O\left(\tau|Y|^{-4}\right)$ as found in (5.14).

Grimshaw [12] considered a slowly varying wave packet propagating towards a critical level. The equations used to describe the motion were obtained by the method of multiple scales by Grimshaw [11]. This slowly varying approximation corresponds to the large Richardson number approximation used here. In the critical layer, it was assumed that nonlinearity dominated over viscosity. It was found that the horizontal velocity (and the wave action density) increased as the critical level was approached, then rapidly decreased to zero in the critical layer. For non-zero viscosity, the wave packet was found to induce a permanent acceleration of the mean flow. This induced mean flow is that due to the wavefront and is not the induced mean velocity (5.20), which is due to the diffusion of vorticity from the critical layer.

\section{Acknowledgement}

The author wishes to acknowledge the support of the Australian Research Grants Scheme under Grant No. 83/15835. 


\section{References}

[1] P. Baldwin and P. H. Roberts, "The critical layer in stratified shear flow", Mathematika 17 (1970)102-119.

[2] J. R. Booker and F. P. Bretherton, "The critical layer for internal gravity waves in a shear flow", J. Fluid Mech. 27 (1967)513-539.

[3] S. N. Brown, A, S. Rosen and S. A. Maslowe, "The evolution of a quasi-steady critical layer in a stratified viscous shear flow", Proc. Roy. Soc. London. Ser. A 375 (1981)271-293.

[4] S. N. Brown and K. Stewartson, "On the nonlinear reflexion of a gravity wave at a critical level, Part I", J. Fluid Mech. 100 (1980)577-595.

[5.] S. N. Brown and K. Stewartson, "On the nonlinear reflexion of a gravity wave at a critical level, Part II", J. Fluid Mech. 115 (1982)217-230.

[6] S. N. Brown and K. Stewartson, "On the nonlinear reflexion of a gravity wave at a critical level, Part III", J. Fluid Mech. 115 (1982)231-250.

[7] T. J. Dunkerton, "Wave transcience in a compressible atmosphere. Part II; Transient equatorial waves in a quasi-biennial oscillation", J. Atmospheric Sci. 38 (1981)281-297.

[8] T. J. Dunkerton, "Wave transcience in a compressible atmosphere, Part III: The saturation of internal gravity waves in the mesosphere", J. Atmospheric Sci. 39 (1982)1042-1051.

[9] T. J. Dunkerton and D. C. Fritts, "Transient gravity wave-critical level interaction Part I: Convective adjustment and the mean zonal acceleration", J. Atmospheric Sci. 41 (1984)992-1007.

[10] D. C. Fritts and T. J. Dunkerton, "A quasi-linear study of gravity wave saturation and self-acceleration", J. Atmospheric Sci. 41 (1984)3272-3289.

[11] R. Grimshaw, "Internal gravity waves in a slowly varying, dissipative medium", Geophys. Fluid Dyn. 6 (1974)131-148.

[12] R. Grimshaw, "Nonlinear internal gravity waves and their interaction with the mean wind", J. Atmospheric Sci. 32 (1975) 1779-1793.

[13] R. Grimshaw, "Wave action and wave-mean flow interaction, with application to stratified shear flows", Ann. Rev. Fluid Mech. 16 (1984)11-44.

[14] P. Hazel, "The effect of viscosity and heat conduction on internal gravity waves at a critical level", J. Fluid Mech. 30 (1967)775-783. 